\title{
Research on Power Quality Analysis and Detecting System
}

\author{
Zhiqiang Zhao ${ }^{1,2}$ \\ 1. State Grid Xinjiang Electric Power Corporation, China \\ 2. North China Electric Power University, China \\ tdqiushipaper@163.com
}

Keywords: power quality; on-line monitoring; power system

\begin{abstract}
With the extensive use of modern power electronic devices and non-linear devices, the problem of pollution of power quality is getting worse. The improvement of power quality assurance has become an important issue faced by the electricity sector. It is for this reason that power quality monitoring and analysis has become a topic of great significance. This paper concisely presents a research on the distributed power quality monitoring system based on digital signal processor (DSP). The system is able to perform regional real-time monitoring of power quality, which is of prime importance to the power sector in enhancing the supervision of power quality and also improve grid operation as well as management level.
\end{abstract}

\section{Introduction}

Electric energy is one of the important energy, with transportation safety, economical, easy to use, clean and easy to control and conversion, is the main driving force of industrial production, providing high-quality and safe and stable electricity power sector is the main task, as the converter equipment and non-linear loads so that the grid power quality is deteriorating, brought to power system such as voltage fluctuation and flicker, harmonics, voltage unbalance three-phase power quality problems.

Power quality problems is an important research direction of power system operation. There are a Effective monitoring of power quality has the dual effect of economic and technology. Not only for the power grid and the operation mode is optimized to achieve economic operation and energy-saving effect. Also provide power quality control measures and data to support decision-making basis, and improve investment than power quality governance. Power quality monitoring of grid security, reliable and economic operation of great significance.

\section{Power Quality Indicators and Detection Algorithm}

2.1 Definition of Power Quality. Power quality refers to the supply of electricity sector client's power quality. Ideally the grid is fixed frequency sinusoidal voltage and standards. In the three-phase AC system, the amplitude of each phase voltage and current should be equal size, symmetry and phase difference of $120^{\circ}$. Since the power systems of generators, transformers and line equipment is nonlinear or asymmetrical, incomplete and run operation, foreign interference and control means various failures and other reasons, resulting in energy pollution. In fact over power condition does not exist, also produced the concept of power quality.

\subsection{The Main Indicators of Power Quality}

Voltage tolerance. Voltage is one of the important indexes of power quality. The voltage quality of power system security and economic operation, to ensure user safety in production and product quality and safety of electrical equipment has important influence. Voltage deviation is refers to the 
power system voltage allowed slowly changing power supply when the deviation of the measured voltage to the rated voltage. Its computation as formula (1)

$$
V_{d}(\%)=\frac{U_{r e}-U_{N}}{U_{N}} \times 100 \%
$$

$U_{r e}$ to the actual voltage value, $U_{N}$ to the nominal voltage.

Voltage fluctuations and flicker. Effective voltage amplitude of voltage fluctuation is regular or random variations within a certain range. Is a series of voltage fluctuation or voltage variation continuous bias voltage, the voltage amplitude variation is typically $90 \%$ to $110 \%$ rating. Voltage fluctuation value $\Delta U$ is the rms voltage of the extremes $U_{\max }$, and the difference between the $U_{\min }$, often with voltage fluctuation value and the nominal voltage $U_{N}$ expressed as a percentage relative voltage fluctuation $d$ as formula (2) .

$$
d=\frac{U_{\max }-U_{\min }}{U_{N}} \times 100 \%
$$

Voltage Flicker refers to the human eye is illuminated by the abnormal voltage fluctuations caused by generating visual experience. The amount of light as incandescent usually pass judgment. Flicker factors, including fluctuations in supply voltage, a lighting device and human visual sensitivity and the like. Flicker can be divided into periodic and aperiodic two, the former is mainly due to voltage fluctuations caused by cyclical, such as compressor, electric arc lamp.

Power harmonic. Harmonic refers to a periodic exchange amount Fourier series decomposition to obtain a frequency of the fundamental frequency component of an integer multiple greater than one. In the power system, typically using harmonic ratio HR and total harmonic distortion THD to reflect the harmonic content and the degree of influence on the voltage and current.

Harmonic ratio refers to the periodic AC component, the first $\mathrm{h}$ times than the fundamental component RMS and RMS of the harmonic component. H-harmonic voltage content ratio $H R U_{h}$, as formula (3).

$$
H R U_{h}=\frac{U_{h}}{U_{1}} \times 100 \%
$$

THD is the ratio of the amount of the periodic exchange of the harmonic content of the effective value of its effective value of the fundamental component, expressed as a percentage, the total voltage distortion rate is $T H D_{u}, T H D_{u}$ as formula (4).

$$
T H D_{u}=\frac{\sqrt{\sum_{h-2}^{M} u_{h}^{2}}}{U_{1}} \times 100 \%
$$

Three-phase voltage unbalance. In the three-phase power supply system, symmetrical threephase voltage is the same as the definition of each phase voltage and current frequency, amplitude and equal phase difference of 120 degrees. Three-phase sequence component vector has a positive and negative sequence components. Phase imbalance refers to the three-phase power system threephase voltage unbalance degree. Negative sequence voltage component of positive sequence component rms voltage unbalance percentage degree [1][2].

\section{Design of Power Quality Monitoring System Platform}

3.1 Power Quality Monitoring System. The main function of power quality monitoring devices functions described here include: peripheral signal conditioning circuits and A / D converter circuit 
acquisition of three-phase voltage and current signals, DSP realization analysis of power quality parameters, access to energy parameters, and power quality monitoring and upload, including frequency deviation, voltage deviation, voltage fluctuation, three-phase voltage unbalance and harmonics. The block diagram of power quality monitoring system is shown in Fig.1.

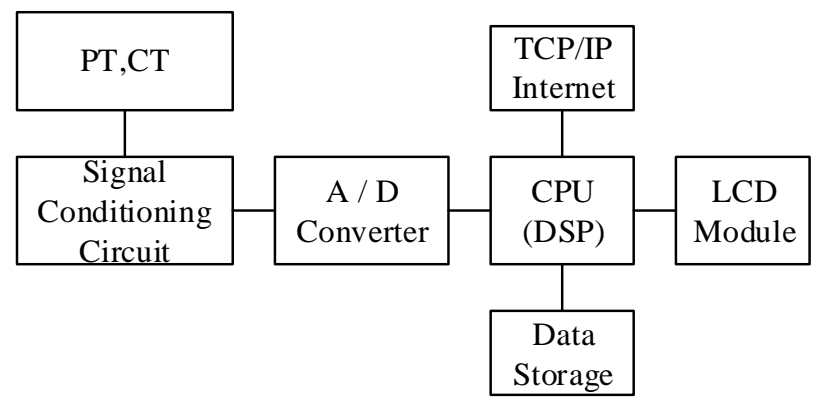

Figure. 1 The block diagram of power quality monitoring system

Transmission line transformer secondary voltage and current signals through the signal conditioning circuit, anti-aliasing filter link the signal phase and amplitude adjustment. A / D conversion circuit under control of the DSP, analog signal analog to digital conversion of the last DSP sampling data processing and analysis, obtained the power quality indicators detected. In order to facilitate local observations, monitoring the voltage parameters through the LCD display, system design features power quality parameters [3].

3.2 Data Processor. The data processor is the core of power quality monitoring system. Power System contamination is random, all systems monitor power quality should have real-time. When large amounts of data online calculation, if the ordinary CPU chip to achieve these operations can not be done in real time. DSP is a high-performance floating-point digital signal processors. It improves execution speed, is ideal for real-time online data collection calculation processing to ensure that the device has a very high accuracy. Power Quality Monitoring System data processing chip used DSP, The DSP model is TMS320F2812.

3.3. Data Communication. In order to achieve the power system energy measurement at different locations, and centralized data analysis. Remote centralized monitoring and analyzing power quality establishment. This design of power quality monitoring system, increasing the embedded TCP / IP protocol. Communication TCP / IP and is responsible for remote management between the data and statistics collected will promptly transferred to the main station management unit, enabling supply of power quality monitoring system in all regions. To achieve a centralized monitoring a plurality of different power systems, improve the degree of power system distribution automation [4].

\section{Design of Power Quality Monitoring Software System}

The role of the main program is a system initialization settings, data acquisition and processing tasks. The results are processed and sent through the distal end of the communication module. The flow chart is shown in Fig. 2.

The data processing is mainly for signal acquisition processing, running a variety of algorithms for the power quality and other parameters calculation and analysis. Discrete signals through fast Fourier transform, it can be isolated from the fundamental component and harmonic components. And changes in the use of FFT result information contained in the voltage deviation can be achieved, the power frequency and phase unbalance calculation [5]. 


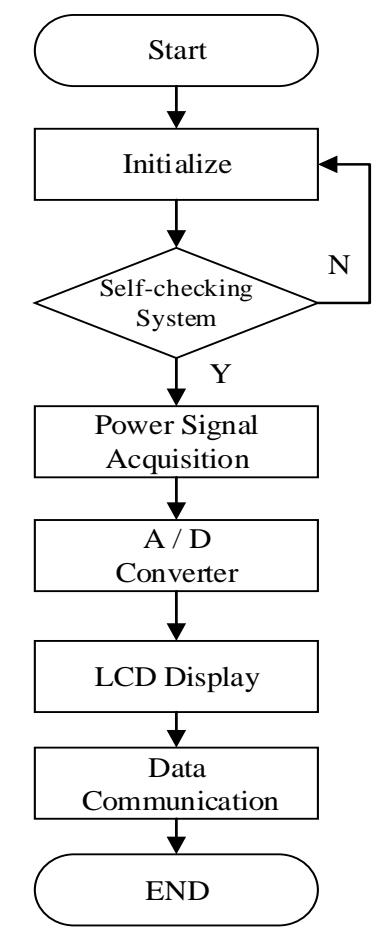

Figure.2 The flow chart of software

\section{Conclusions}

In this paper, the parameters and the measurement principles of electric energy parameters are studied, the power quality monitoring system is designed, the hardware and software systems were introduced, real-time power quality monitoring system for grid power is explained as well as the timely detection of problems in power quality. The system is beneficial in carrying out smooth monitoring and control of power quality to promote the improvement in network security and economic operation.

\section{References}

[1]Jian Q.Z, Power Quality Analysis and Monitoring Techniques [D],Shandong,Shandong University,2005.

[2]Xun X.B, The Research of Distributed Power Quality on-line Monitoring System [D],Henan, Henan Polytechnic University,2014.

[3] Akagi H, Kanazawa Y, Nabae A. Instantaneous reactive Power compensators comprising switching devices without energy storage components [J]. IEEE Transactions on Industry Applications 1984, 20(3): 625-630.

[4] AngrisaniL, Daponte P. A measurement method based on the Wavelet Transform for PowerQuality Analysis[J].IEEE Transactions on Power Delivery, 1998, 13 (4) :990-998.

[5] Pal Yash and A. SWARUP Bhim Singh, "Flexible Control of Single-phase Unified Power Quality Conditioner Using Frequency-domain Approach", Electric Power Components and Systems, vol. 41, pp. 401-412, 2013 